\title{
Increasing prevalence of diabetic ketoacidosis at diabetes diagnosis among children in Quebec: a population-based retrospective cohort study
}

\author{
Marie-Eve Robinson MD MSc, Patricia Li MD MSc, Elham Rahme PhD, Marc Simard MSc, \\ Isabelle Larocque MSc, Meranda M. Nakhla MD MSc
}

\section{Abstract}

Background: Diabetic ketoacidosis at type 1 diabetes diagnosis is a preventable life-threatening complication. Canadian data on the temporal trends of the prevalence of diabetic ketoacidosis at the onset of type 1 diabetes in children are unknown. We aimed to determine the temporal changes in diabetic ketoacidosis prevalence at diabetes diagnosis in Quebec.

Methods: We conducted a population-based cohort study of children (aged 1-17 yr) living in Quebec who were diagnosed with diabetes between 2001 and 2014, using multiple health administrative linked databases available at the Institut national de santé publique du Québec through the Quebec Integrated Chronic Surveillance System. We used multivariate Poisson regression analysis with robust error variance to determine trends in the prevalence of diabetic ketoacidosis.

Results: We found that $25.6 \%(1471 / 5741)$ of children presented with diabetic ketoacidosis at diabetes diagnosis. The incidence of diabetes was stable at 30 cases per 100000 children per year during the study period. The age- and sex-standardized rates of diabetic ketoacidosis increased from 22\% (95\% confidence interval [Cl] 17\%-26\%) in 2001 to 30\% (95\% Cl 24\%-36\%) in 2014. The relative increase of diabetic ketoacidosis prevalence at diabetes diagnosis over the study period was $2.0 \%$ per year (rate ratio 1.02 ; $95 \%$ Cl 1.01-1.03).

Interpretation: Despite a stable incidence of type 1 diabetes, we found that the prevalence of diabetic ketoacidosis at diabetes onset increased between 2001 and 2014. Our findings are concerning and demonstrate a need to continue to campaign to recognize type 1 diabetes before diabetic ketoacidosis supervenes.

1 ype 1 diabetes (T1D) is one of the most common chronic diseases of childhood and is associated with significant morbidity and mortality. ${ }^{1,2}$ Its incidence in children and adolescents is increasing by $3 \%-5 \%$ per year worldwide. In Canada, the incidence of T1D is among the highest in the world (32 cases per 100000 children and adolescents per year). ${ }^{1-5}$ The diagnosis of T1D is preceded by typical symptoms of polyuria, polydipsia, weight loss and fatigue. If T1D is left undiagnosed and untreated, diabetic ketoacidosis (DKA) develops. DKA is an acute, avoidable, life-threatening complication and the leading cause of preventable hospital admissions, emergency department visits and deaths in the T1D population. ${ }^{1,6,7}$ The economic costs of 1 DKA hospital admission are high, ranging from US\$4125 to US\$11 $196 .^{8}$ In addition to the risk of acute morbidity, DKA is associated with long-term morbidity. Recent studies suggest that DKA at diagnosis predicts poor long-term glycemic control, independent of demographic and socioeconomic factors. . $^{9,10}$
Globally, DKA rates at T1D diagnosis vary between $12 \%$ and $80 \%$ and are inversely correlated with the population incidence of T1D, suggesting that increased awareness of T1D results in earlier diagnosis and treatment, leading to decreased DKA risk. ${ }^{11,12}$ Despite a global increase in the incidence of T1D, most regions have reported stable rates of DKA. ${ }^{13-18}$ Other countries, such as Italy and Finland, have reported decreasing DKA rates at diabetes diagnosis, coinciding with diabetes awareness programs. ${ }^{19,20}$ In the United States, temporal trends in DKA rates at diabetes diagnosis

Competing interests: Meranda Nakhla received a lecture fee from the Quebec Association of Endocrinologists. No other competing interests were declared.

This article has been peer reviewed.

Correspondence to: Meranda Nakhla, meranda.nakhla@mcgill.ca CMAJ Open 2019. DOI:10.9778/cmajo.20190047 
have been conflicting. ${ }^{13,21}$ The SEARCH for Diabetes in Youth Study, a multicentre registry of youth with diabetes in the US, reported stable temporal trends in DKA prevalence at diabetes diagnosis over 3 time periods (2002-2003, 20042005 and 2008-2010). ${ }^{13}$ Colorado, on the other hand, demonstrated increasing DKA rates at T1D diagnosis between 1998 and 2012. ${ }^{21}$ The population burden of DKA may underscore gaps in health care access and/or in knowledge of the early symptoms of T1D among the lay and medical community. To supply Canadian data on the temporal trends of DKA prevalence at T1D onset in children, we aimed to examine the temporal trends in DKA prevalence at T1D diagnosis among children and adolescents in Quebec, from 2001 to 2014.

\section{Methods}

\section{Study design}

We conducted a population-based retrospective cohort study of children (aged 1-17 yr) living in Quebec who were diagnosed with diabetes between Apr. 1, 2001, and Mar. 31, 2014. Quebec is Canada's second-largest province, with over 8 million residents, and it has a universal public health insurance coverage system through which all residents are insured for medically necessary health care services. As such, all encounters with the health care system are contained within the provincial health administrative data.

\section{Data sources}

We used multiple health administrative databases of all people with diabetes available at the Institut national de santé publique du Québec (INSPQ) through the Quebec Integrated Chronic Surveillance System (QICDSS). The QICDSS includes the Registered Persons Database (patient demographics), the Physician Service Claims Database (physician remunerated services across all clinical settings) and the Hospital Discharge Database (MED-ECHO). The Régie de l'assurance maladie du Québec linked these databases using a unique individual encoded identifier.

\section{Cohort identification}

We identified our cohort using the validated definition of the Canadian Chronic Disease Surveillance System (CCDSS) to identify diabetes within administrative data. This definition has been shown to have a $99.9 \%$ specificity and $94.2 \%$ sensitivity in identifying people less than 18 years of age with diabetes within Canadian health administrative databases. ${ }^{22}$ This case definition requires 1 hospital admission, or 2 physician visit claims for diabetes in 2 years. The date of diagnosis of diabetes was the first physician visit claim or the first hospital admission coded for diabetes, whichever occurred first. Although DKA can occur at diagnosis in both T1D and type 2 diabetes (T2D) in children, ${ }^{23}$ the CCDSS definition does not distinguish between T1D and T2D. In Canada, the incidence of T1D is 32 cases per 100000 children and youth and the incidence of non-T1D (including T2D) is 2.3 cases per 100000 children and youth per year. ${ }^{24,25}$ Patients with invalid health insurance numbers were excluded.

\section{Outcome}

Our primary outcome was DKA at the time of a diabetes diagnosis. We identified DKA episodes diagnosed within 3 days of the date of diagnosis of diabetes taking place during outpatient or emergency department visits or during a hospital admission using the Physician Service Claims Database (Quebec version of the International Classification of Diseases, 9th Revision [ICD9], codes 250.1-250.2) and the MED-ECHO hospital discharge database (ICD-9 codes 250.1-250.2 before 2006 and Canadian version of the International Statistical Classification of Diseases and Related Health Problems, 10th Revision, codes E10.10-E14.10 and E10.12-E14.12 thereafter). We chose a 3-day window as this was considered a reasonably short period wherein a patient was probably in DKA at the time of diagnosis. ${ }^{26}$ These DKA codes have been used in previous studies in children to identify DKA within Canadian health administrative databases, ensuring that outcomes were measured consistently and using the same criteria. ${ }^{26-28}$

\section{Patient characteristics at diagnosis}

The following patient characteristics at diagnosis (Registered Persons Database) were included: age at diabetes diagnosis, sex, calendar year, combined material and social deprivation index (neighbourhood level proxy for socioeconomic status) ${ }^{29}$ and rural status. Age was categorized into the following groups: 1-4 years, 5-11 years and 12-17 years, representing preschool age, school age and adolescence, respectively. These age groups have been previously shown to have differing risks associated with DKA at diagnosis. ${ }^{13,26}$ The material and social deprivation index and rural status were assigned from the person's residential postal code at diabetes diagnosis. The material deprivation index is based on employment, education level and income. The social deprivation index is based on the proportion of people living alone and the proportion of single-parent families. ${ }^{29}$ The material and social deprivation index is a validated index for Canada derived by the INSPQ on the basis of census dissemination areas. ${ }^{29}$ To obtain this index, our study cohort was divided across quintiles of their combined material and social deprivation indices $(\mathrm{Q} 1$ = least deprived, Q5 = most deprived). The combined material and social quintiles were then grouped into 3 categories: least deprived (Q1-Q2), moderately deprived (Q3) and most deprived (Q4-Q5). Rural status was defined as urban (population > 100000 ), small cities (population 10 000-100 000) and rural (population < 10 000).

\section{Statistical analysis}

To compare DKA prevalence at diabetes diagnosis by year, we used direct standardization to control for differences in age distribution and stratified by sex (standardized to 2001 Quebec age distribution). We used $t$ tests to compare continuous variables and the Pearson $\chi^{2}$ test to compare categorical variables between those that presented in DKA and those that did not. Multivariate Poisson regression analyses with robust error variance were used to model the relationship between calendar year and DKA prevalence at diabetes onset, adjusted for sex, age group at diagnosis, rural status and socioeconomic status. ${ }^{30}$ Subanalyses were performed using multivariate Poisson 
regression analyses with robust error variance to model the relationship between calendar year and DKA prevalence at disease onset, for each age group and adjusted for sex, rural status and socioeconomic status. People with missing variables were included. Statistical tests were 2 -sided with a significance level of $p<0.05$. Analyses were performed using SAS version 9.4 (SAS Institute, Cary, NC).

\section{Sensitivity analysis}

We conducted a sensitivity analysis whereby we included only DKA episodes requiring a hospital admission in the multivariate Poisson regression analysis to model the relationship between calendar year and first-onset DKA requiring a hospital admission, adjusted for sex, age group, rural status and socioeconomic status.

\section{Ethics approval}

The study was approved by the McGill University Health Centre Research Ethics Board.

\section{Results}

We identified 5741 new cases of diabetes among children and adolescents between Apr. 1, 2001, and Mar. 31, 2014. The mean age at diagnosis was 10.1 years (standard deviation $4.8 \mathrm{yr})$ and $52.1 \%$ were boys. Overall, 1471 children $(25.6 \%$ of the study population) presented with DKA at diabetes diagnosis (Appendix 1, available at www.cmajopen.ca/content/7/2/ E300/suppl/DC1). The crude proportions and adjusted rate ratio of DKA at diabetes diagnosis by age, sex, socioeconomic status and rural status are shown in Table 1.

Over the study period, the incidence of diabetes remained stable at 30 cases per 100000 children per year (Figure 1). Ageand sex-standardized DKA prevalence at diabetes diagnosis increased from $22.0 \%$ (95\% confidence interval [CI] $17.3 \%-$ $27.7 \%$ ) in 2001 to $29.6 \%$ (95\% CI $23.8 \%-37.1 \%$ ) in 2014 (Appendix 1 and Figure 1). In the multivariate analysis, the relative increase in DKA was $2.0 \%$ per year (rate ratio $1.02 ; 95 \%$ CI $1.01-1.03, p$ for trend $<0.001)$. Children diagnosed with diabetes in 2014 were $38.6 \%$ more likely to present with DKA than those diagnosed in 2001 (rate ratio 1.39 , 95\% CI 1.081.79) (Appendix 1). The relative annual increase in DKA prevalence among children aged 1-4 years, 5-11 years and 12-17 years was $0.2 \%$ (rate ratio 1.002 ; 95\% CI 0.976-1.029), $2.7 \%$ (rate ratio $1.027 ; 95 \%$ CI $1.009-1.045$ ) and $2.3 \%$ (rate ratio 1.023; 95\% CI 1.004-1.042), respectively. Overall, children aged $12-17$ years were $30.1 \%$ less likely to present with DKA than children aged $1-4$ years (rate ratio $0.70,95 \%$ CI $0.62-$ 0.79 ), while children living in small cities were $16.0 \%$ more likely to present with DKA than children living in urban settings (rate ratio 1.16, 95\% CI 1.02-1.32) (Table 1). There was no association between socioeconomic status and DKA risk.

\section{Sensitivity analysis}

Among the 1471 children who presented with DKA at diabetes diagnosis, 1402 were admitted to hospital. The relative annual increase in the number of children with DKA

\begin{tabular}{|c|c|c|}
\hline Characteristic & $\begin{array}{l}\text { No. }(\%) \text { of } \\
\text { patients with } \\
\text { DKA, by } \\
\text { characteristic }\end{array}$ & $\begin{array}{l}\text { Adjusted rate } \\
\text { ratio }(95 \% \mathrm{Cl})^{*}\end{array}$ \\
\hline \multicolumn{3}{|l|}{ Age group, yr } \\
\hline $1-4$ & $296(29.1)$ & 1.00 \\
\hline $5-11$ & $659(30.1)$ & $1.03(0.92-1.16)$ \\
\hline $12-17$ & $526(20.5)$ & $0.70(0.62-0.79)$ \\
\hline \multicolumn{3}{|l|}{ Sex } \\
\hline Male & $793(26.5)$ & 1.00 \\
\hline Female & $678(24.7)$ & $0.96(0.92-1.01)$ \\
\hline \multicolumn{3}{|l|}{ SES† } \\
\hline Least deprived & $597(24.7)$ & 1.00 \\
\hline Moderately deprived & $295(26.5)$ & $1.09(0.97-1.23)$ \\
\hline Most deprived & $528(26.1)$ & $1.06(0.96-1.18)$ \\
\hline \multicolumn{3}{|l|}{ Rural status $\ddagger$} \\
\hline Urban, population > 100000 & $956(25.2)$ & 1.00 \\
\hline Small cities, 10 000-100 000 & 205 (29.2) & $1.16(1.02-1.32)$ \\
\hline Rural, $<10000$ & $294(24.4)$ & $0.95(0.85-1.06)$ \\
\hline \multicolumn{3}{|c|}{$\begin{array}{l}\text { Note: } \mathrm{Cl}=\text { confidence interval, DKA = diabetic ketoacidosis, SES = } \\
\text { socioeconomic status. } \\
\text { *Poisson regression was conducted to adjust for age group, sex, socioeconomic } \\
\text { status, rural status and year. } \\
\text { †SES was derived from combined social and material deprivation indices. Data } \\
\text { on SES were missing for } 189 \text { children: } 138 \text { without DKA and } 51 \text { with DKA. Social } \\
\text { and material quintiles were combined into a } 5 \times 5 \text { table, and the } 25 \text { combined } \\
\text { categories were grouped into } 3 \text { categories: least deprived, moderately deprived } \\
\text { and most deprived. } \\
\text { fData on rural status were missing for } 48 \text { children: } 32 \text { without DKA and } 16 \text { with } \\
\text { DKA. }\end{array}$} \\
\hline
\end{tabular}

requiring hospital admission over the study period was still significant at $1.2 \%$ per year (rate ratio 1.012 , 95\% CI $1.001-1.023, p$ for trend $<0.05)$.

\section{Interpretation}

In this population-based study, over a quarter of children presented in DKA at diabetes onset. DKA prevalence at diagnosis increased between 2001 and 2014 from 22\% to 30\%, representing a relative increase of $2 \%$ per year, despite the fact that the incidence of diabetes was stable during that period. We continued to observe a relative annual increase in DKA prevalence over time, when DKA episodes not requiring admission were excluded (presumably representing milder cases). We found that DKA risk was higher among younger than older children, and it was higher among children living in small cities than among those living in urban areas. Our results suggest that an increasing number of children are experiencing delays in the diagnosis and treatment of diabetes.

Colorado is the only other region that has reported increasing temporal trends in DKA prevalence at diabetes diagnosis; in that state, among 3439 children with T1D, 


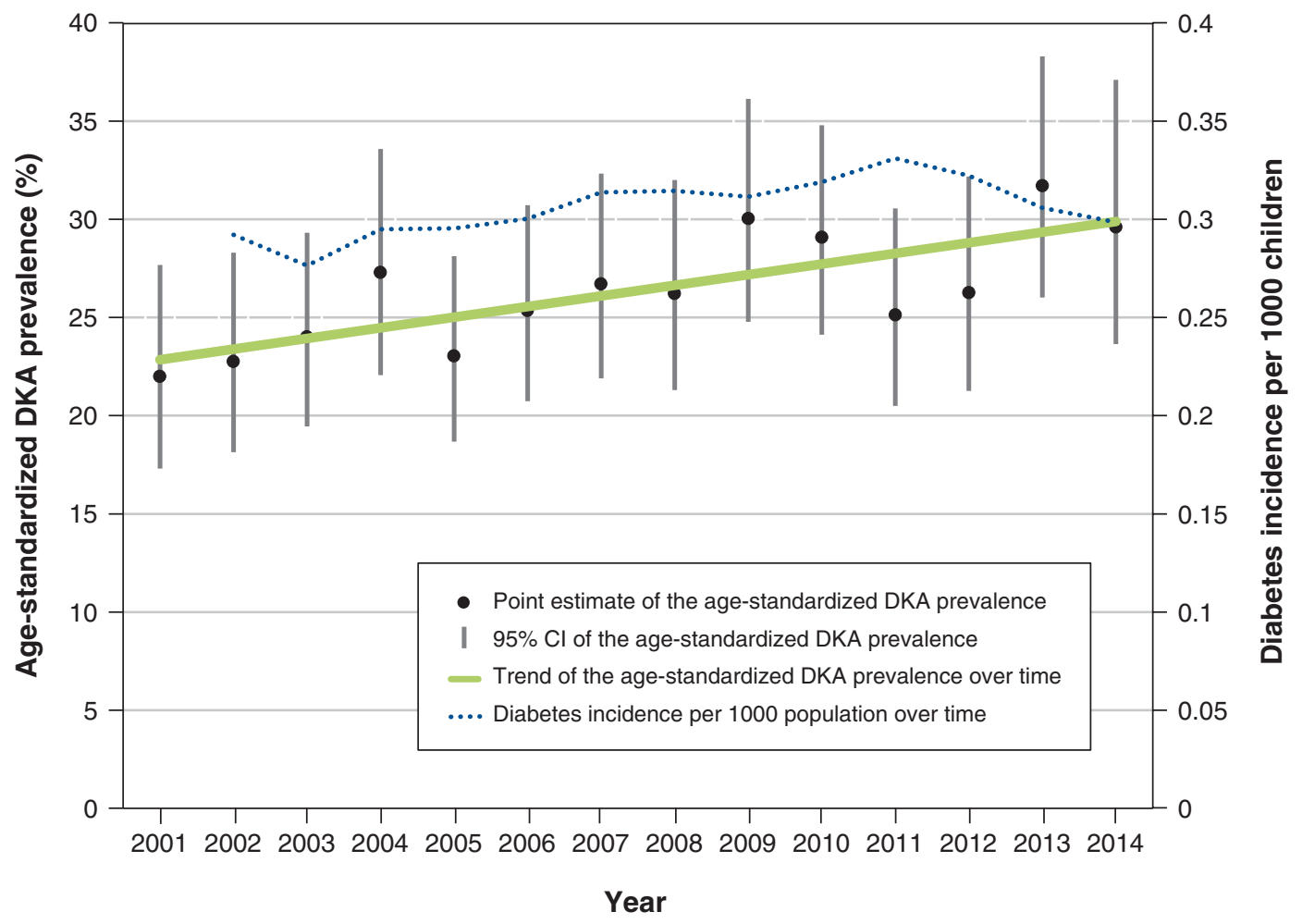

Figure 1: Prevalence of diabetic ketoacidosis at diabetes onset over time. Note: $\mathrm{Cl}=$ confidence interval, DKA = diabetic ketoacidosis. The agestandardized DKA prevalence increased over time among 5741 children with diabetes (from $22 \%$ [95\% Cl 17\%-26\%] in 2001 to 30\% [95\% Cl $24 \%-36 \%]$ in 2014).

$38.9 \%$ had DKA at T1D diagnosis and the prevalence of DKA at diagnosis increased by $55 \%$ (from $29.9 \%$ to $46.2 \%$ ) between 1998 and 2012. ${ }^{21}$ The US SEARCH for Diabetes in Youth Study reported stable temporal trends in DKA prevalence at diabetes diagnosis from 2002 to $2010 .{ }^{13}$ That study's prevalence of DKA at diagnosis was similar to ours, wherein approximately one-third of youth presenting with new-onset T1D were in DKA. ${ }^{13}$ In Germany, among 14664 patients with T1D, DKA prevalence at diagnosis was $21.1 \%$; however, despite a $4.4 \%$ annual increase in T1D incidence, temporal trends in DKA prevalence at diabetes diagnosis remained stable (1995-2007). ${ }^{16}$ Austria has also reported stable temporal trends in DKA prevalence at diabetes diagnosis (1998-2008), with a DKA prevalence somewhat higher than observed in our population at $37.2 \% .{ }^{17}$ Other countries, such as Finland, have reported declines in DKA prevalence at diagnosis with rates of $18.9 \%$ in $1982-1991$ compared with $15.2 \%$ in 1992 $2001(p=0.028) .{ }^{19}$

The reasons for the increasing trend we observed are unclear. In Colorado, the increasing trends in DKA were partially attributed to the increasing prevalence of child poverty in that state. In our study, we did not find any association between socioeconomic status and DKA risk, which may be explained by Canada's universal-coverage health care system. However, the DKA prevalence at diagnosis in our study is high and is increasing. Limited and deteriorating access to primary health care in Canada may be a contributing factor. We have previously documented that $41 \%$ of children with newly diagnosed T1D living in Quebec did not have a usual provider of primary care and that having a primary care provider was associated with a reduced DKA risk at diagnosis in children older than 5 years of age. ${ }^{26}$ Furthermore, wait times for urgent primary care visits are longer in Canada than in other countries and continue to increase ${ }^{31}$ Lack of physician and public awareness of the signs and symptoms of T1D may be another contributor to the increasing trends in DKA. In Finland, increasing physician and public awareness through 2 mechanisms may arguably have contributed to the observed decrease in DKA prevalence at diagnosis. ${ }^{19}$ First, Finland has one of the highest incidence rates of T1D in the world, such that public and physician awareness of the symptoms and signs are high, and the diagnosis is often made before DKA develops. Second, a T1D prediction and prevention trial was carried out in the Finish population, whereupon newborn infants carrying HLA-conferred susceptibility to T1D were invited to participate in regular follow-up to detect possible 
signs of diabetes, plausibly increasing awareness. ${ }^{32}$ In addition, studies in other countries evaluating educational campaigns have shown that when public and physician awareness of the symptoms of diabetes increases, DKA rates at diagnosis decrease. ${ }^{20}$ As in our study, younger age has consistently been identified as a risk factor for DKA at diabetes onset, including in a systematic review of 46 studies that identified younger age, particularly under 2 years, as a risk factor for DKA at diagnosis. ${ }^{33}$ Several factors may account for this increased DKA risk, including the following: (a) physicians may have a lower index of suspicion for T1D in younger children, (b) younger children may have a faster metabolic decompensation, (c) younger children may not be able to verbalize their diabetes symptoms and (d) their symptoms may be more subtle and not as apparent as in older children, leading to delayed diagnosis and treatment. ${ }^{34-36}$ These factors may also explain why we found that DKA risk did not increase over time among young children, as these are factors, other than physician awareness, that are not modified by time or interventions. Our finding of a higher risk of DKA among children living in small cities than among with those living in urban settings is in contrast to European data wherein no association between DKA risk and urban/rural status had been found. ${ }^{33}$ Our observations could be explained in part by differing distributions of physician supply and access across regions. ${ }^{37}$ This could not be confirmed in our study and requires further exploration.

\section{Limitations}

Although our study population was restricted to 1 province, access to primary care is suboptimal across all provinces and thus our study probably provides a generalizable representation of DKA rates at diagnosis across Canada. ${ }^{26,31}$ The diagnosis of DKA was based on administrative data and not on biochemical grounds; as such, we could not distinguish between the varying severities of DKA. Further, we could not adjust for other factors associated with DKA such as ethnicity or family history of diabetes, because these are not captured in provincial administrative databases. ${ }^{38,39}$ Using health administrative databases, we could not distinguish between T1D and T2D; nevertheless, approximately $95 \%$ of children younger than 18 years of age with diabetes have T1D. ${ }^{40}$ Given that DKA can occur in both forms of diabetes, our finding of increasing trends of DKA at diagnosis is a concerning observation irrespective of the type of diabetes the child has, highlighting the importance of determining the underlying drivers.

\section{Conclusion}

In this large population-based study, we have demonstrated increasing temporal trends in DKA prevalence at diabetes diagnosis despite a stable incidence of diabetes in Quebec. Our results are concerning and underscore the need to increase physician and public awareness of the signs and symptoms of childhood diabetes before DKA develops and to impress upon physicians the importance of referring children immediately. For instance, an educational prevention program in Parma, Italy, directed at physicians, teachers and the public saw a significant decrease in the prevalence of DKA at diabetes diagnosis. ${ }^{12,20}$ As other countries have demonstrated stable or decreasing trends in DKA prevalence, future research, potentially through international collaborations, should investigate the reasons for our increasing DKA trends, to develop targeted and effective interventions.

\section{References}

1. Dahlquist G, Kallen B. Mortality in childhood-onset type 1 diabetes: a population-based study. Diabetes Care 2005;28:2384-7.

2. Edge JA, Ford-Adams ME, Dunger DB. Causes of death in children with insulin dependent diabetes 1990-96. Arch Dis Child 1999;81:318-23.

3. Fox DA, Islam N, Sutherland J, et al. Type 1 diabetes incidence and prevalence trends in a cohort of Canadian children and youth. Pediatr Diabetes 2018;19:501-5.

4. Nakhla M, Rochette L, Rahme E, et al. Trends in diabetes prevalence and incidence in children and youth in Quebec, Canada 2000-2008: a populationbased study. Proceedings of the 37th ISPAD Annual Conference; 2011 Oct. 19-22; Miami. Berlin: International Society of Pediatric and Adolescent Diabetes.

5. Patterson CC, Dahlquist GG, Gyürüs E, et al.; EURODIAB Study Group. Incidence trends for childhood type 1 diabetes in Europe during 1989-2003 and predicted new cases 2005-20: a multicentre prospective registration study. Lancet 2009;373:2027-33.

6. Glasgow AM, Weissberg-Benchell J, Tynan WD, et al. Readmissions of children with diabetes mellitus to a children's hospital. Pediatrics 1991;88:98-104.

7. Keenan HT, Foster CM, Bratton SL. Social factors associated with prolonged hospitalization among diabetic children. Pediatrics 2002;109:40-4.

8. Tieder JS, McLeod L, Keren R, et al.; Pediatric Research in Inpatient Settings Network. Variation in resource use and readmission for diabetic ketoacidosis in children's hospitals. Pediatrics 2013;132:229-36.

9. Duca LM, Wang B, Rewers M, et al. Diabetic ketoacidosis at diagnosis of type 1 diabetes predicts poor long-term glycemic control. Diabetes Care 2017;40:1249-55.

10. Shalitin S, Fisher S, Yackbovitch-Gavan M, et al. Ketoacidosis at onset of type 1 diabetes is a predictor of long-term glycemic control. Pediatr Diabetes 2018;19:320-8.

11. Jefferies CA, Nakhla M, Derraik JG, et al. Preventing diabetic ketoacidosis. Pediatr Clin North Am 2015;62:857-71.

12. King BR, Howard NJ, Verge CF, et al. A diabetes awareness campaign prevents diabetic ketoacidosis in children at their initial presentation with type 1 diabetes. Pediatr Diabetes 2012;13:647-51.

13. Dabelea D, Rewers A, Stafford JM, et al.; SEARCH for Diabetes in Youth Study Group. Trends in the prevalence of ketoacidosis at diabetes diagnosis: the SEARCH for diabetes in youth study. Pediatrics 2014;133:e938-45.

14. de Vries L, Oren L, Lebenthal Y, et al. Decrease in frequency of ketoacidosis at diabetes onset over the past two decades - perspectives of a paediatric tertiary care centre. Diabet Med 2012;29:e170-5.

15. Jefferies C, Cutfield SW, Derraik JG, et al. 15-year incidence of diabetic ketoacidosis at onset of type 1 diabetes in children from a regional setting (Auckland, New Zealand). Sci Rep 2015;5:10358.

16. Neu A, Hofer SE, Karges B, et al.; DPV Initiative and the German BMBF Competency Network for Diabetes Mellitus. Ketoacidosis at diabetes onset is still frequent in children and adolescents: a multicenter analysis of 14,664 patients from 106 institutions. Diabetes Care 2009;32:1647-8.

17. Schober E, Rami B, Waldhoer T; Austrian Diabetes Incidence Study Group. Diabetic ketoacidosis at diagnosis in Austrian children in 1989-2008: a population-based analysis. Diabetologia 2010;53:1057-61.

18. Trellu M, Lacombe S, Morin D, et al. Epidemiology of diabetes in children in Languedoc-Roussillon (France) [article in French]. Arch Pediatr 2015; 22:241-6.

19. Hekkala A, Knip M, Veijola R. Ketoacidosis at diagnosis of type 1 diabetes in children in northern Finland: temporal changes over 20 years. Diabetes Care 2007;30:861-6.

20. Vanelli M, Chiari G, Ghizzoni L, et al. Effectiveness of a prevention program for diabetic ketoacidosis in children. An 8-year study in schools and private practices. Diabetes Care 1999;22:7-9.

21. Rewers A, Dong F, Slover RH, et al. Incidence of diabetic ketoacidosis at diagnosis of type 1 diabetes in Colorado youth, 1998-2012. FAMA 2015;313:1570-2.

22. Dart AB, Martens PJ, Sellers EA, et al. Validation of a pediatric diabetes case definition using administrative health data in Manitoba, Canada. Diabetes Care 2011;34:898-903.

23. Klingensmith GJ, Connor CG, Ruedy KJ, et al.; Pediatric Diabetes Consortium. Presentation of youth with type 2 diabetes in the Pediatric Diabetes Consortium. Pediatr Diabetes 2016;17:266-73.

24. Amed S, Dean HJ, Panagiotopoulos C, et al. Type 2 diabetes, medicationinduced diabetes, and monogenic diabetes in Canadian children: a prospective national surveillance study. Diabetes Care 2010;33:786-91. 
25. Guttmann A, Nakhla M, Henderson M, et al. Validation of a health administrative data algorithm for assessing the epidemiology of diabetes in Canadian children. Pediatr Diabetes 2010;11:122-8.

26. Nakhla M, Rahme E, Simard M, et al. Risk of ketoacidosis in children at the time of diabetes mellitus diagnosis by primary caregiver status: a populationbased retrospective cohort study. CMA7 2018;190:E416-21.

27. Nakhla M, Rahme E, Simard M, et al. Outcomes associated with a pediatric clinical diabetes network in Ontario: a population-based time-trend analysis. CMA7 Open 2017;5:E586-93.

28. Shulman R, Stukel TA, Miller FA, et al. Low socioeconomic status is associated with adverse events in children and teens on insulin pumps under a universal access program: a population-based cohort study. BM7 Open Diabetes Res Care 2016;4:e000239.

29. Pampalon R, Hamel D, Gamache P, et al. An area-based material and social deprivation index for public health in Québec and Canada. Can 7 Public Health 2012;103(Suppl 2):S17-22.

30. Blais C, Jean S, Sirois C, et al. Quebec Integrated Chronic Disease Surveillance System (QICDSS), an innovative approach. Chronic Dis Inj Can 2014;34:226-35.

31. Health care in Canada, 2012: a focus on wait times. Ottawa: Canadian Institute for Health Information; 2012.

32. Kupila A, Muona P, Simell T, et al. Juvenile Diabetes Research Foundation Centre for the Prevention of Type I Diabetes in Finland. Feasibility of genetic and immunological prediction of type I diabetes in a populationbased birth cohort. Diabetologia 2001;44:290-7.

33. Usher-Smith JA, Thompson MJ, Sharp SJ, et al. Factors associated with the presence of diabetic ketoacidosis at diagnosis of diabetes in children and young adults: a systematic review. BMF 2011;343:d4092.

34. Bui H, To T, Stein R, et al. Is diabetic ketoacidosis at disease onset a result of missed diagnosis? 7 Pediatr 2010;156:472-7.

35. Komulainen J, Kulmala P, Savola K, et al. Clinical, autoimmune, and genetic characteristics of very young children with type 1 diabetes. Childhood Diabetes in Finland (DiMe) Study Group. Diabetes Care 1999;22:1950-5.

36. Sochett EB, Daneman D, Clarson C, et al. Factors affecting and patterns of residual insulin secretion during the first year of type 1 (insulin-dependent) diabetes mellitus in children. Diabetologia 1987;30:453-9.

37. Guttmann A, Shipman SA, Lam K, et al. Primary care physician supply and children's health care use, access, and outcomes: findings from Canada. Pediatrics 2010;125:1119-26.

38. Choleau C, Maitre J, Filipovic Pierucci A, et al.; AJD Study Group. Ketoacidosis at diagnosis of type 1 diabetes in French children and adolescents. Diabetes Metab 2014;40:137-42.

39. Duca LM, Reboussin BA, Pihoker C, et al. Diabetic ketoacidosis at diagnosis of type 1 diabetes and glycemic control over time: the SEARCH for diabetes in youth study. Pediatr Diabetes 2019;20:172-9.
40. Shulman R, Miller FA, Stukel TA, et al. Resources and population served: a description of the Ontario Paediatric Diabetes Network. CMA7 Open 2016;4:E141-6

Affiliations: Divisions of Endocrinology (Robinson, Nakhla) and General Pediatrics (Li), Department of Pediatrics, McGill University Health Centre; Research Institute of the McGill University Health Centre (Li, Rahme, Nakhla), Montréal, Que.; Institut national de santé publique du Québec (Simard, Larocque), Québec, Que.

Contributors: Marie-Eve Robinson contributed to the study conception and design, oversaw the analysis and interpreted the data. Elham Rahme, Isabelle Larocque, Patricia Li and Meranda Nakhla conceptualized and designed the study, oversaw the analysis and interpreted the data. Marc Simard contributed to the study conception and design, carried out the analysis and contributed to the interpretation of the data. Marie-Eve Robinson drafted the initial manuscript and all other authors critically revised it for important intellectual content. All authors approved the final manuscript for publication and agreed to be accountable for all aspects of the work.

Funding: Meranda Nakhla was funded by a Chercheur-boursier clinicien Junior 2 award from the Fonds de recherche du Québec - Santé (FRQS) and the Ministère de la Santé et des Services sociaux du Québec. Patricia $\mathrm{Li}$ was funded by a Chercheur-boursier clinicien Junior 1 award from the FRQS and the Ministère de la Santé et des Services sociaux du Québec and a New Investigator Salary Award from the Canadian Institutes of Health Research (CIHR). This research was supported by Meranda Nakhla's and Patricia Li's grants from the FRQS and CIHR. The funders played no role in the conduct or management of the study; the collection analysis or the interpretation of data; or the preparation of the manuscript

Acknowledgement: The authors acknowledge the contributions of Dr. Denis Daneman (professor and chair emeritus, Department of Paediatrics, University of Toronto; paediatrician-in-chief emeritus, Hospital for Sick Children) in critically reviewing the manuscript.

Supplemental information: For reviewer comments and the original submission of this manuscript, please see www.cmajopen.ca/content $/ 7 / 2$ / E300/suppl/DC1. 